\title{
Original article (short paper) \\ Hypotensive effects of resistance exercise with continuous and intermittent blood flow restriction
}

\author{
Gabriel Rodrigues Neto \\ Universidade Federal de Paraíba, João Pessoa, Paraíba, Brasil \\ Universidade Federal do Rio de Janeiro, Rio de Janeiro, RJ, Brasil \\ Jefferson da Silva Novaes \\ Universidade Federal do Rio de Janeiro, Rio de Janeiro, RJ, Brasil \\ Michel Gonçalves \\ Universidade Federal do Rio de Janeiro, Rio de Janeiro, RJ, Brasil \\ Instituto de Pesquisa e Capacitação Física do Exército, Rio de Janeiro, RJ, Brasil
}

Gilmário Ricarte Batista

Rosa Maria Soares Costa de Mendonça

Universidade Federal da Paraíba, João Pessoa, PB, Brasil

Humberto Miranda

Universidade Federal do Rio de Janeiro, Rio de Janeiro, RJ, Brasil

Giovanni da Silva Novaes

Universidade Castelo Branco, Rio de Janeiro, RJ, Brasil

Centro de Investigação em Esporte, Saúde e Desenvolvimento Humano, Vila Real, Portugal

Maria do Socorro Cirilo-Sousa

Universidade Federal da Paraíba, João Pessoa, PB, Brasil

\begin{abstract}
The aim of this study was to compare the acute effects of low-intensity (LI) resistance exercise (RE) with continuous blood flow restriction (CBFR) and intermittent blood flow restriction (IBFR) on systolic blood pressure (SBP), diastolic blood pressure (DBP), and mean arterial pressure (MAP). After a one-repetition maximum test, 10 normotensive recreationally trained men performed three experimental protocols. In the three RE protocols, increases in SBP, DBP, and MAP were observed immediately after exercise, but the effect sizes (ESs) were greater for the LI + CBFR and high-intensity protocols. There were hypotensive effects on SBP, DBP, and MAP in all three protocols; however, the effects on MAP lasted longer for the LI + IBFR and LI + CBFR protocols. These long-lasting hypotensive effects on DBP and MAP occurred in all three protocols. Thus, we conclude that the post exercise hypotensive effects on SBP, DBP, and MAP appear to occur in all three RE protocols, with the effect on SBP being longer in the LI + IBFR and LI + CBFR protocols.
\end{abstract}

Keywords: kaatsu, vascular occlusion, hypotension, resistance training

\section{Introduction}

Traditional resistance exercises (REs) have been used to promote post exercise hypotension, which occurs as a result of the reduced vascular resistance observed in these exercise protocols ${ }^{1-3}$. The reduced vascular resistance is caused by an increase in the endothelial substances and by reductions in the sympathetic nervous system activity and cardiac output ${ }^{4}$. In addition, RE enhances the development and maintenance of strength, muscular endurance, power, and muscle hypertrophy ${ }^{5,6}$. However, some individuals cannot be subjected to high-intensity (HI) RE due to several health problems, and therefore, cannot train with heavy loads ( $>70 \%$ one-repetition maximum [1RM]), which are recommended for increasing strength and hypertrophy ${ }^{5}$. Accordingly, low-intensity (LI) RE combined with blood flow restriction (BFR) can be used for these individuals because 
this method uses low loads (20-30\% 1RM) combined with a tourniquet, which reduces the negative effect of heavy loads on the joints ${ }^{7,8}$. The strength and muscle mass gains obtained with this method seem to be as effective as HI training ${ }^{9,10}$; in addition, this method seems to be safe in terms of hemodynamics ${ }^{11-15}$.

Few studies have evaluated potential hypotensive effects [systolic blood pressure (SBP), diastolic blood pressure (DBP), and/or the mean arterial pressure (MAP)] after the application of LI RE with continuous blood flow restriction (CBFR) ${ }^{16,17}$ and intermittent blood flow restriction (IBFR) ${ }^{12,18}$ alone. Among these studies, only three have demonstrated hypotensive responses after LI RE combined with BFR ${ }^{12,16,17}$. However, only Brandner, Kidgell, and Warmington ${ }^{19}$ compared the hypotensive effect of CBFR and IBFR RE (unilateral elbow flexion exercise trials). They found no post exercise hypotension and presented some limitations that may have affected the results, such as the amount of exercises used, how they were performed (unilaterally or single-jointed), and the different pressures used for the BFR. In addition, there are few studies showing similar effects when CBFR and IBFR RE were compared in terms of muscle activation, strength, and lean mass ${ }^{20,21}$. While both have benefits for strength and hypertrophy, the best strategy for working with BFR, either in a single exercise or in an exercise session, remains unknown. Thus, it would be interesting to understand the stimuli in blood pressure to verify the degree of change and observe what would be the safest method of BFR (continuous vs. intermittent) in a RE session with BFR. However, it is necessary to conduct studies in healthy populations (e.g., youth and adults) with safe procedures and methods appropriate to later be extrapolated in special populations.

A literature search revealed that no study to date has included analysis of the hypotensive response after a RE session for the upper limbs with CBFR and IBFR. Accordingly, we hypothesized that the hypotensive response observed after HI exercises and after LI RE with CBFR and IBFR would be similar. Therefore, the aim of this study was to compare the acute effects of LI RE with CBFR and IBFR on SBP, DBP, and MAP in normotensive recreationally trained men.

\section{Methods}

\section{Subjects}

In total, 10 normotensive recreationally trained military men $(19 \pm 0.8$ years old; $78.8 \pm 10.8 \mathrm{~kg} ; 174.6 \pm 5.4 \mathrm{~m} ; 25.7 \pm 2.7$ $\mathrm{m}^{2} \cdot \mathrm{kg}^{-1}$ ) aged $18-21$ years performed the three experimental protocols in random order. Those considered recreationally trained had between 1 and 5 years of experience in strength training ${ }^{22}$. The sample was selected by convenience; however, the subjects included should be normotensive men aged between 18 and 30 years. The subjects were randomly divided to undergo the three protocols after selection (crossover design). The exclusion criteria were (1) individuals who responded "yes" to any of the Physical Activity Readiness Questionnaire items ${ }^{23}$, (2) individuals who missed one RE session, (3) individuals who had some type of musculoskeletal injury of the upper limbs, and (4) smokers. After being informed of the risks and benefits of the study, the subjects signed an informed consent form prepared in accordance with the principles of the Declaration of Helsinki. The study was approved by the local ethics committee (Protocol 0476/13).

\section{Study Design}

Anthropometric parameters and muscle strength were assessed during the first visit to the laboratory. After this visit, the subjects visited the laboratory on three different occasions 72-96 hours apart. During these visits, the subjects completed the three protocols in random order (crossover design): (1) LI RE (20\% 1RM) combined with IBFR (LI + IBFR), (2) LI RE (20\% 1RM) combined with CBFR (LI + CBFR), and (3) HI RE (80\% 1RM). All three protocols were performed at the same time of day to control diurnal blood pressure variation. Blood pressure was measured before (resting); immediately after; and then 15, 30, 45, and 60 min after performing the RE protocols. Subjects were instructed to avoid exhausting exercise, caffeine and chocolate intake, use of nutritional supplements, and intake of alcoholic beverages during and after the effort sessions and were advised to sleep at least 6 hours in the night before the exercise session. In addition, subjects were instructed to maintain the same eating habits during the study period and not to perform the Valsalva maneuver during all RE sessions.

\section{Procedures}

\section{Anthropometric Assessment}

Height and body mass were measured to the nearest $0.5 \mathrm{~cm}$ and $0.1 \mathrm{~kg}$, respectively. A stadiometer and a balance scale (Filizola scale, model 31; Sao Paulo, SP, Brazil) were used in the study.

\section{RM Test}

The exercises were performed bilaterally and included the bench press, lat pulldown, triceps extension, and biceps curl. The subjects performed a warm-up set of 5-10 repetitions at 40-60\% of perceived maximum with a 1-min rest interval between the sets. After the 1-min rest interval, the second set was performed with three to five repetitions at $60-80 \%$ of the perceived maximum. The strength evaluation started after a 1-min rest interval and measurement within five attempts. The load was adjusted before each new attempt. Rest intervals between the attempts were standardized at 3-5 min, with 20 min elapsed between different exercises. The test was discontinued when the subject failed to properly perform the movement, and the load moved in the last successful attempt was then considered the maximum load. The following strategies were adopted to reduce the margin of error in data collection procedures: (1) all tests were performed at the same time of day in each session; (2) standardized instructions were given before the test so that each subject was aware of the entire data collection routine; (3) the subject was instructed regarding the proper technique to perform the exercise; and (4) all subjects received standardized verbal encouragement during the tests. 


\section{Assessment of Blood Pressure}

Before and after each RE session, blood pressure was measured using a stethoscope (3M Littmann ${ }^{\circledR}$ Cardiology; 3M, St. Paul, MN, USA) and an aneroid sphygmomanometer (DuraShock $^{\mathrm{TM}}$ Tycos $^{\circledR}$, Model DS-44; Welch Allyn, Skaneateles Falls, NY). The cuff was completely wrapped around the right arm, enclosing at least two-thirds of the upper arm. Blood pressure flow measurements were performed $15 \mathrm{~min}$ immediately before (two measures resting); immediately after exercise (post-30-45 $\mathrm{s}$ ); and approximately $15 \mathrm{~min}$ (post-15), $30 \mathrm{~min}$ (post-30), 45 min (post-45), $60 \mathrm{~min}$, and more than $60 \mathrm{~min}$ (post-60) after each protocol. Immediately after the exercise session, the subject was instructed to sit, then the standard tourniquet was removed and a sphygmomanometer was placed for blood pressure measurement. All measurements were performed by the same expert evaluator according to the guidelines of the American Heart Association ${ }^{24}$. MAP was calculated according to the equation

$$
(\mathrm{SBP}+2 \mathrm{DBP}) / 3
$$

\section{Determination of $B F R$}

After the assessment of blood pressure using the sphygmomanometer, subjects remained seated and a standard sphygmomanometer was placed in the axillary fold region to measure blood pressure (Riester Komprimeter pneumatic tourniquet for hemostasis in the extremities) in the arm (width of $60 \mathrm{~mm}$ and length of $470 \mathrm{~mm}$ ) to verify blood pressure before starting each protocol of CBFR and IBFR. The total BFR was set at 1.3 times resting $\mathrm{SBP}^{14,19,25}$. The cuff was inflated and deflated between sets. The mean pressures used throughout the entire exercise protocols of IBFR and CBFR were $163.8 \pm 10.5 \mathrm{mmHg}$ and $160.9 \pm 12.9 \mathrm{mmHg}$, respectively.

\section{Experimental Sessions}

Four bilateral REs were performed, including the bench press (with conventional bar and calibrated rings), lat pull-down, triceps extension, and biceps curl (on conventional machines). Subjects performed three protocols in random order: four LI REs (20\% 1RM) combined with IBFR (LI + IBFR), four LI REs (20\% 1RM) combined with CBFR (LI + CBFR), and four HI RE (80\% 1RM). For the LI RE protocols with CBFR and IBFR, the subjects completed 30 repetitions followed by 3 sets of 15 repetitions ( $20 \% 1 \mathrm{RM})$, with an interval of $30 \mathrm{~s}$ between all sets and a 1-min interval between exercises while wearing a standard sphygmomanometer that was placed on the upper arm (proximal; width $60 \mathrm{~mm}$ and length $470 \mathrm{~mm}$ ) for blood pressure measurement (Riester Komprimeter pneumatic tourniquet for hemostasis in the extremities). The cuff was deflated between sets for the LI + IBFR protocol and maintained inflated between sets for the LI + CBFR protocol. For the HI protocol, subjects completed three sets of eight repetitions ( $80 \% 1 \mathrm{RM})$ with 2 -min rest intervals between the sets and a 1-min rest interval between exercises. The velocity of the movement was controlled by a metronome set at $3 \mathrm{~s}(1.5 \mathrm{~s}$ for the concentric muscular action and $1.5 \mathrm{~s}$ for the eccentric muscular action).

\section{Total Work Exercises}

The total work (TW) performed was calculated by multiplying the total load, the sets, and the complete repetitions of the four exercises for each protocol (load $\times$ sets $\times$ repetitions).

\section{Statistical Analysis}

The statistical analysis was initially performed using the Shapiro-Wilk test for normality and Levene's test for homogeneity of variances. The variables were normally distributed and homogeneous $(p>0.05)$. The test of reproducibility was performed between the first and second measurements of blood pressure at rest before starting each protocol (intraclass correlation coefficient [ICC]). A paired $t$-test was used to compare the two BFR pressures used in the LI + IBFR and LI + CBFR protocols, and one-way analysis of variance (ANOVA) with the Bonferroni post hoc test was used to compare TW between protocols. Two-way repeated-measures ANOVA (protocols [LI + IBFR vs. LI + CBFR vs. HI] $\times$ time [resting vs. immediately after exercise vs. $15 \mathrm{~min}$ vs. $30 \mathrm{~min}$ vs. $45 \mathrm{~min}$ vs. $60 \mathrm{~min}]$ ) followed by Bonferroni's post hoc comparisons test was used to analyze the possible differences in SBP, DBP, and MAP. The ES was used to verify the magnitude (trivial $<0.35$, small $0.35-0.80$, moderate $0.80-1.50$, and large $>1.5$ ) of changes between assessments of the protocols ${ }^{22}$, and the percentage change $(\Delta \%)$ was used to express the possible differences between the significant changes. The level of significance was set at $p \leq 0.05$. All statistical analyses were performed using the IBM SPSS version 20.0 statistical software package (IBM, Armonk, NY).

\section{Results}

The blood pressure ICCs calculated by the researcher were as follows: $\mathrm{LI}+\mathrm{IBFR}=0.975, \mathrm{LI}+\mathrm{CBFR}=0.966$, and $\mathrm{HI}=0.959$. All subjects completed the three exercise sessions. The paired $t$-test revealed no significant differences between the two BFR pressures used in the LI + IBFR vs. LI + CBFR protocol ( $p=0.444)$. Regarding TW (bench press, lat pulldown, triceps extension, and biceps curl), there was a significant difference between the LI vs. HI RE protocol (LI + IBFR and LI + CBFR [4131.0 \pm 608.2$]$ vs. HI $[5598.7 \pm 836.7], p<0.001)$; however, there was no significant difference between LI + IBFR and LI + CBFR $(p=1.000)$.

In the comparative analysis of SBP, significant differences were found between LI + CBFR and HI at 15 and 30 min ( $p$ $=0.017$ and $p=0.050$, respectively). A significant increase in SBP between resting and immediately after the exercises in all protocols was observed (LI + IBFR, $p<0.001, \Delta \%=24.6 \%$, $\mathrm{ES}=3.05 ; \mathrm{LI}+\mathrm{CBFR}, p<0.001, \Delta \%=35.7 \%, \mathrm{ES}=5.62$; and HI, $p<0.001, \Delta \%=35.2 \%, \mathrm{ES}=3.80)$. Significant reductions 
were observed between resting and $45 \min (p=0.021, \Delta \%=$ $-6.8 \%, \mathrm{ES}=0.85)$ and between resting and $60 \mathrm{~min}(p=0.003$, $\Delta \%=-8.5 \%, \mathrm{ES}=1.05)$ in the LI + IBFR protocol. For the LI + CBFR protocol, a significant reduction was observed between resting and $60 \min (p=0.003, \Delta \%=-8.3 \%, \mathrm{ES}=1.31)$. In the HI protocol, significant reductions were observed between resting and $15 \min (p=0.005, \Delta \%=-8.1 \%, \mathrm{ES}=0.88)$ and between resting and $30 \min (p=0.022, \Delta \%=-7.3 \%, \mathrm{ES}=0.79)$ (Table 1). In the LI + CBFR protocol, a significant reduction was observed between resting and $60 \min (p=0.003, \Delta \%=$ $-8.3 \%, \mathrm{ES}=1.31)$. For the HI protocol, significant reductions were observed between resting and $15 \min (p=0.005, \Delta \%=$ $-8.1 \%, \mathrm{ES}=0.88)$ and between resting and $30 \mathrm{~min}(p=0.022$, $\Delta \%=-7.3 \%, \mathrm{ES}=0.79)$ (Table 1$)$.

In the comparative analysis of DBP, significant increases were observed between resting and immediately after exercise in all protocols (LI + IBFR, $p<0.001, \Delta \%=16.6 \%, \mathrm{ES}=1.26$; LI $+\mathrm{CBFR}, p<0.001, \Delta \%=15.7 \%, \mathrm{ES}=1.73$; and $\mathrm{HI} p<0.001$, $\Delta \%=22.7 \%, E S=2.46)$. Significant reductions were observed between resting and $60 \mathrm{~min}$ in all protocols (LI + IBFR, $p=$ $0.004, \Delta \%=-10.8 \%, \mathrm{ES}=0.82 ; \mathrm{LI}+\mathrm{CBFR}, p=0.001, \Delta \%$ $=-12.7 \%, \mathrm{ES}=1.47$; and HI, $p=0.014, \Delta \%=-8.8 \%$, ES $=$ 0.95 ), as shown in Table 2.

In the comparative analysis of MAP, a significant difference was observed between LI + CBFR and AI at $15 \mathrm{~min}(p=$ 0.033 ). The analysis of the different times revealed significant increases between resting and immediately after exercise in all protocols (LI + IBFR, $p<0.001, \Delta \%=20.3 \%, \mathrm{ES}=2.03$; $\mathrm{LI}+\mathrm{CBFR}, p<0.001, \Delta \%=24.4 \%, \mathrm{ES}=3.48$; and HI, $p<$ $0.001, \Delta \%=28.1 \%, \mathrm{ES}=3.16)$. Significant reductions were observed between resting and $60 \min (p=0.001, \Delta \%=-9.7 \%$, $\mathrm{ES}=0.96)$ in the LI + IBFR protocol. For the LI + CBFR protocol, there were significant reductions between resting and $60 \min (p<0.001, \Delta \%=-10.7 \%, \mathrm{ES}=1.52)$. For the HI protocol, there were significant reductions between resting and $15 \min (p=0.013, \Delta \%=-7.1 \%, \mathrm{ES}=0.80)$ and between resting and $60 \min (p=0.008, \Delta \%=-7.1 \%, \mathrm{ES}=0.80)$, as shown in Table 3.

Table 1. Comparative analysis of systolic blood pressure in different study protocols.

\begin{tabular}{|c|c|c|c|c|c|c|}
\hline \multirow[b]{2}{*}{ Protocols } & \multicolumn{6}{|c|}{ Systolic Blood Pressure (mm Hg) } \\
\hline & Resting & $\begin{array}{c}\text { Immediately after } \\
\text { exercise }\end{array}$ & 15 minutes & 30 minutes & 45 minutes & 60 minutes \\
\hline $\mathrm{LI}+\mathrm{IBFR}$ & $123.5 \pm 10.0$ & $154.0 \pm 21.7 *$ & $120.0 \pm 12.4$ & $117.0 \pm 12.5$ & $115.0 \pm 11.7 *$ & $113.0 \pm 14.9^{*}$ \\
\hline $\mathrm{LI}+\mathrm{CBFR}$ & $126.0 \pm 8.0$ & $171.0 \pm 17.9^{*}$ & $126.5 \pm 11.5 \dagger$ & $123.5 \pm 12.0 \dagger$ & $120.5 \pm 8.9$ & $115.5 \pm 11.1^{*}$ \\
\hline $\mathrm{HI}$ & $122.0 \pm 11.3$ & $165.0 \pm 15.8^{*}$ & $112.0 \pm 13.9^{*}$ & $113.0 \pm 9.4^{*}$ & $117.0 \pm 9.4$ & $116.0 \pm 9.6$ \\
\hline
\end{tabular}

\footnotetext{
* Significant difference between resting and immediately after exercises, resting and $15 \mathrm{~min}$, resting and $30 \mathrm{~min}$, resting and $45 \mathrm{~min}$, resting and $60 \mathrm{~min}$ Significant difference between $\mathrm{LI}+\mathrm{CBFR}$ and $\mathrm{HI}$

$\mathrm{LI}+\mathrm{IBFR}=$ low-intensity resistance exercises combined with intermittent blood flow restriction; $\mathrm{LI}+\mathrm{CBFR}=$ low-intensity resistance exercises combined with continuous blood flow restriction; $\mathrm{HI}=$ high-intensity resistance exercises
}

Table 2. Comparative analysis of diastolic blood pressure between the study protocols.

\begin{tabular}{ccccccc}
\hline & \multicolumn{5}{c}{ Diastolic Blood Pressure (mm Hg) } \\
\hline Protocols & Resting & $\begin{array}{c}\text { Immediately after } \\
\text { exercise }\end{array}$ & $\mathbf{1 5}$ minutes & $\mathbf{3 0}$ minutes & 45 minutes & $\mathbf{6 0}$ minutes \\
\hline LI + IBFR & $78.0 \pm 10.3$ & $91.0 \pm 8.7^{*}$ & $75.5 \pm 9.5$ & $75.0 \pm 10.8$ & $74.5 \pm 11.6$ & $69.5 \pm 12.1^{*}$ \\
LI + CBFR & $82.5 \pm 7.1$ & $95.5 \pm 6.4^{*}$ & $81.0 \pm 8.7$ & $79.0 \pm 7.3$ & $77.0 \pm 9.4$ & $72.0 \pm 7.5^{*}$ \\
HI & $79.0 \pm 7.3$ & $97.0 \pm 9.4^{*}$ & $74.0 \pm 8.4$ & $78.0 \pm 8.3$ & $78.0 \pm 7.8$ & $72.0 \pm 7.8^{*}$ \\
\hline
\end{tabular}

*Significant difference between resting and immediately after exercise, resting and $60 \mathrm{~min}$

$\mathrm{LI}+\mathrm{IBFR}=$ low-intensity resistance exercises combined with intermittent blood flow restriction; $\mathrm{LI}+\mathrm{CBFR}=$ low-intensity resistance exercises combined with continuous blood flow restriction; $\mathrm{HI}=$ high-intensity resistance exercises.

Table 3. Comparative analysis of mean arterial pressure between the study protocols.

\begin{tabular}{|c|c|c|c|c|c|c|}
\hline \multirow[b]{2}{*}{ Protocols } & \multicolumn{6}{|c|}{ Mean Arterial Pressure (mm Hg) } \\
\hline & Resting & $\begin{array}{l}\text { Immediately } \\
\text { after exercise }\end{array}$ & 15 minutes & 30 minutes & 45 minutes & 60 minutes \\
\hline $\mathrm{LI}+\mathrm{IBFR}$ & $93.1 \pm 9.4$ & $112.0 \pm 12.3^{*}$ & $90.3 \pm 9.7$ & $89.0 \pm 10.3$ & $87.9 \pm 11.2$ & $84.0 \pm 12.3^{*}$ \\
\hline $\mathrm{LI}+\mathrm{CBFR}$ & $96.9 \pm 6.8$ & $120.6 \pm 9.1 *$ & $96.1 \pm 8.8 \dagger$ & $93.8 \pm 7.5$ & $91.4 \pm 8.9$ & $86.5 \pm 7.7 *$ \\
\hline $\mathrm{HI}$ & $93.3 \pm 8.3$ & $119.6 \pm 9.7 *$ & $86.6 \pm 9.6^{*}$ & $89.6 \pm 6.7$ & $90.9 \pm 7.7$ & $86.6 \pm 6.8^{*}$ \\
\hline
\end{tabular}

\footnotetext{
* Significant difference between resting and immediately after exercise, resting and 15 min, resting and 60 min

Significant difference between LI + CBFR and HI

$\mathrm{LI}+\mathrm{IBFR}=$ low-intensity resistance exercises combined with intermittent blood flow restriction; $\mathrm{LI}+\mathrm{CBFR}=$ low-intensity resistance exercises combined with continuous blood flow restriction; $\mathrm{HI}=$ high-intensity resistance exercises.
} 


\section{Discussion}

In this study, we compared the acute effects of RE combined with CBFR and IBFR on SBP, DBP, and MAP in military men. To our knowledge, this study involves the first comparison of the hypotensive responses elicited by upper limb exercises done with CBFR or IBFR. The main findings of this study are (1) the three RE protocols increased SBP, DBP, and MAP immediately after exercises; and (2) hypotensive responses (changes in SBP, DBP, and MAP) were observed in the three protocols without significant differences between them. However, the effects on SBP were longer in the LI+ IBFR and LI + CBFR protocols.

Acute increases in SBP, DBP, and MAP were observed in the three protocols without significant differences between them, but a smaller magnitude was observed in the LI + IBFR protocol than in the other protocols. These increases may have occurred because IBFR promotes lower levels of muscle pain than $\mathrm{CBFR}^{21}$, which could cause less tension on the muscles involved. The acute findings of the study conducted by Brandner, Kidgell, Warmington ${ }^{19}$ comparing CBFR and IBFR were different from those in the present study. Those authors reported a higher magnitude in the LI + IBFR protocol, a result that may have been due to the pressure used in IBFR $(151 \mathrm{mmHg})$, which was much higher than the pressure used in CBFR $(91 \mathrm{mmHg})$. However, other studies corroborate our findings. Previous studies reported increases in blood pressure immediately after application of LI RE combined with CBFR..$^{14,15,26}$ This increase in blood pressure shown in previous studies and in the present study may occur because CBFR potentiates elevations in muscle sympathetic nerve activity, heart rate, and $\mathrm{MAP}^{27}$. In contrast, a previous study has found no significant differences in SBP, DBP, and MAP in the IBFR protocol ${ }^{28}$. This finding may have been due to several factors, including the low pressure of the BFR $(100 \mathrm{mmHg})$ used in their protocol, no vascular occlusion (BFR) between sets, and the use of only two single-joint exercises of the lower limbs.

The HI protocol promoted a faster hypotensive response (SBP and MAP) that started 15 min after the end of the exercises. An explanation of this finding is that the TW of the HI protocol was significantly higher than in other protocols. Indeed, it has been stated that TW plays an important role in the hypotensive respons $\mathrm{e}^{29,30}$. These findings are in agreement with previous studies $^{15-17}$, that detected hypotensive responses after LI RE combined with CBFR or IBFR. In contrast to other studies ${ }^{11,18,19}$.

Therefore, upon analyzing the methodological procedures according to the body segment used in the exercises and the subjects of the studies, it seems that the greatest magnitude of hypotension occurs in the following scenarios: in individuals who performed RE combined with IBFR in the upper and lower limbs (agonist-antagonist) ${ }^{12}$, in hypertensive individuals who performed RE combined with CBFR (lower limbs) ${ }^{16}$, and in apparently healthy young individuals who performed RE combined with CBFR at 40\% 1RM (upper limbs) ${ }^{17}$. However, it was also observed that the hypotensive response did not occur in apparently healthy young subjects who performed LI RE $(\leq 30 \%$ $1 \mathrm{RM}$ ) with CBFR and IBFR in single body segments ${ }^{19}$. On the other hand, loads $\leq 30 \% 1 \mathrm{RM}$ are able to promote hypotensive responses after LI RE with CBFR in hypertensive individuals ${ }^{16}$.
Additionally, it seems that performing exercises for upper and lower limbs in the same session can promote greater hypotensive effects than the effects after exercises performed with only one segment, which can be associated with the amount of muscle mass involved ${ }^{31}$. Thus, the lower blood pressure observed in the present study (using exercises for upper limbs and trunk) may have occurred due to the larger amount of muscle mass involved leading to increased nitric oxide production via endothelial shear stress, which then plays an important role in protecting vascular tone $\mathrm{e}^{32,33}$.

In the study performed by Brandner, Kidgell, Warmington ${ }^{19}$, the first group of investigators who compared the effects of CBFR or IBFR RE (unilateral elbow flexion exercises) on hypotension, no significant hypotensive responses were observed. Some methodological limitations might explain these findings. These authors compared the effects of upper limb RE on hypotensive responses $(5,20,40$, and $60 \mathrm{~min}$ post exercise) in four different conditions: HI ( $80 \%$ 1RM, four sets of six to eight repetitions with an interval of $150 \mathrm{~s}$ between sets), LI without BFR (20\% 1RM, 4 sets, with 30 repetitions in the first set and 15 repetitions in the second, third, and fourth sets; an interval of $30 \mathrm{~s}$ between sets), and two LI protocols with application of either CBFR $(91 \mathrm{mmHg})$ or IBFR $(151 \mathrm{mmHg})$ using a cuff with a width of $10.5 \mathrm{~cm}$. The use of only a single-joint exercise (arm flexion) that was performed unilaterally and at different pressures for CBFR ( $80 \%$ resting SBP) and IBFR (130\% resting SBP) might explain the lack of hypotensive responses in both BFR protocols. Because of the methodological procedures used, these results are not similar to the results of the present study, where bilateral and multi-joint exercises and not significantly different pressures for CBFR and IBFR were used. Another point worth mentioning is that our training volume was greater than the one used by Brandner, Kidgell, Warmington ${ }^{19}$. In addition, our study has good practical application because four exercises were used, which is similar to a training session.

The faster reduction in SBP observed in the LI + IBFR protocol may have occurred due to the release of the cuff between the sets, which may have promoted reactive hyperemia and shear stress more often than CBFR. These factors, in turn, could increase the production of nitric oxide synthase, the enzyme responsible for the conversion of $\mathrm{L}$-arginine to nitric oxide, a small, electrically neutral molecule capable of promoting positive changes in the endothelium ${ }^{35}$, but with less discomfort ${ }^{21}$ and lower blood pressure changes, as observed in the present study. This protocol can be an excellent option for hypertensive individuals, as the increase in muscle activation ${ }^{20}$ and gains in muscle strength and lean body mass ${ }^{21}$ are similar between CBFR and IBFR. In addition, Takano ${ }^{14}$ mentioned that the decrease in venous return promoted by the RE performed with BFR causes a reduction in cardiac preload during exercise, which may be an excellent strategy for people with vascular diseases.

\section{Conclusions}

The post exercise hypotensive responses occurred in all three protocols (changes in SBP, DBP, and MAP), with longer effects 
on SBP observed in the LI + IBFR and LI + CBFR protocols, although they were faster in the HI protocol. The LI + IBFR protocol appears to have a smaller effect than the other protocols in increasing SBP, DBP, and MAP immediately after exercise. Therefore, it is important to conduct further experiments in normotensive and hypertensive subjects to address both acute and chronic hypotensive responses, particularly involving different BFR pressures and different cuff sizes.

\section{References}

1. Bentes CM, Costa PB, Rodrigues Neto G, Costa e Silva GV, De Salles BF, Miranda HL, Novaes JS. Hypotensive effects and performance responses between different resistance training intensities and exercise orders in apparently health women. Clin Physiol Functional Imaging. 2015; 35:185-190.

2. Matos DG, Aidar FJ, Filho MLM, Silva SR, Oliveira JC, Klain IP, Dantas EHM. Analysis of hemodynamic responses to resistance exercise performed with different intensities and recovery intervals. Health. 2013; 5:159-165.

3. Mohebbi H, Rahmaninia F, Vatani DFH. Post-resistance exercise hypotensive responses at different intensities and volumes. Physical Education Sport. 2009; 7:171-179.

4. Polito MD, Farinatti PTV. Blood pressure behavior after counter-resistance exercises: a systematic review on determining variables and possible mechanisms. Revista Brasileira de Medicina do Esporte. 2006; 12:386-392.

5. ACSM. American College of Sports Medicine. Progression models in resistance training for healthy adults. Medicine Sci Sports Exercise. 2009; 41:687-708.

6. Pollock ML, Franklin BA, Balady GJ, Chaitman BL, Fleg JL, Fletcher B, et al. Resistance exercise in individuals with and wihout cardiovascular disease: benefits, rationale, safety, and prescriptionan. An Advisory From the Committee on Exercise, Rehabilitation, and Prevention, Council on Clinical Cardiology, American Heart Association. Circulation. 2000; 101:828-833.

7. Loenneke JP, Wilson JM, Marín PJ, Zourdos MC, Bemben MG. Low intensity blood flow restriction training: a meta-analysis. Eur J Appl Physiol. 2012; 112: 1849-1859.

8. Scott BR, Loenneke JP, Slattery KM, Dascombe BJ. Exercise with blood flow restriction: an updated evidence-based approach for enhanced muscular development. Sports Medicine. 2015; 45:313-325.

9. Laurentino GC, Ugrinowitsch C, Roschel H, Aoki MS, Soares AG, Neves J, et al. Strength training with blood flow restriction diminishes myostatin gene expression. Medicine Sci in Sports Exercise. 2012; 44:406-412.

10. Takarada Y, Takazawa H, Sato Y, Takebayashi S, Tanaka Y, Ishii N. Effects of resistance exercise combined with moderate vascular occlusion on muscular function in humans. J Appl Physiol. 2000; 88:2097.

11. Fahs CA, Rossow LM, Seo D, Loenneke JP, Sherk VD, Kim E, et al. Effect of different types of resistance exercise on arterial compliance and calf blood flow. European J Appl Physiol. 2011; 111:2969-2975.
12. Neto GR, Sousa MSC, Costa PB, Salles BF, Novaes GS, Novaes JS. Hypotensive effects of resistance exercises with blood flow restriction. J Strength Conditioning Res. 2015; 29:1064-1070.

13. Neto GR, Sousa MSC, Silva GVC, Gil ALS, Salles BF, Novaes JS. Acute resistance exercise with blood flow restriction effects on heart rate, double product, oxygen saturation and perceived exertion. Clin Physiol Functional Imaging. 2016; 36:53-59.

14. Takano H, Morita $\mathrm{T}$, Iida $\mathrm{H}$, Asada $\mathrm{K}$, Kato M, Uno K, et al. Hemodynamic and hormonal responses to a short-term low-intensity resistance exercise with the reduction of muscle blood flow. Eur J Appl Physiol. 2005; 95:65-73.

15. Vieira PJC, Chiappa GR, Umpierre D, Stein R, Ribeiro JP. Hemodynamic responses to resistance exercise with restricted blood flow in young and older men. J Strength Conditioning Res. 2013; 27:2288-2294.

16. Araújo JP, Silva ED, Silva JCG, Souza TSP, Lima EO, Guerra I, et al. The acute effect of resistance exercise with blood flow restriction with hemodynamic variables on hypertensive subjects. J Human Kinetics. 2014; 43:79-85.

17. Maior AS, Simão R, Rocha MMS, Freitas SB, Willardson JM. Influence of blood flow restriction during low-intensity resistance exercise on the post-exercise hypotensive response. J Strength Conditioning Res. 2015: 29:2894-2899.

18. Rossow LM, Fahs CA, Sherk VD, Seo D, Bemben DA, Bemben MG. The effect of acute blood-flow-restricted resistance exercise on postexercise blood pressure. Clin Physiol Functional Imaging. 2011; 31:429-434.

19. Brandner CR, Kidgell DJ, Warmington SA. Unilateral bicep curl hemodynamics: Low-pressure continuous vs high-pressure intermittent blood flow restriction. Scand J Medicine Sci Sports. 2014.

20. Yasuda T, Loenneke JP, Ogasawara R, Abe T. Influence of continuous or intermittent blood flow restriction on muscle activation during low-intensity multiple sets of resistance exercise. Acta Physiol Hung. 2013; 100:419-426.

21. Fitschen PJ, Kistler BM, Jeong JH, Chung HR, Wu PT, Walsh MJ, et al. Perceptual effects and efficacy of intermittent or continuous blood flow restriction resistance training. ClinPhysiol Functional Imaging. 2014; 34:356-363.

22. Rhea MR. Determining the magnitude of treatment effects in strength training research through the use the effect size. J Strength Conditioning Res. 2004; 18: 918-920.

23. Shephard RJ. PAR-Q, Canadian Home Fitness Test and exercise screening alternatives. Sports Medicine. 1988; 5:185-195.

24. Pickering TG, Hall JE, Appel LJ, Falkner BE, Graves J, Hill $\mathrm{MN}$, et al. Recommendations for blood pressure measurement in humans and experimental animals part 1: blood pressure measurement in humans: a statement for professionals from the Subcommittee of Professional and Public Education of the American Heart Association Council on High Blood Pressure Research. Circulation. 2005; 111:697-716.

25. Suga T, Okita K, Morita N, Yokota T, Hirabayashi K, Horiuchi $\mathrm{M}$, et al. Dose effect on intramuscular metabolic stress during low-intensity resistance exercise with blood flow restriction. J Appl Physiol. 2010; 108:1563-1567.

26. Poton R, Polito MD. Cardiovascular responses during resistance exercise with blood flow restriction. Revista Brasileira de Cardiologia. 2014; 27: 104-110. 
27. Victor RG, Seals DR. Reflex stimulation of sympathetic outflow during rhythmic exercise in humans. Am J Physiol. 1989; 257:H2017-H2024.

28. Figueroa A, Vicil F. Post-exercise aortic hemodynamic responses to low-intensity resistance exercise with and without vascular occlusion. Scandinavian J Medicine Sci Sports. 2011; 21:431-436.

29. Mediano MFF, Paravidino V, Simão R, Pontes FL, Polito MD. Subacute behavior of the blood pressure after power training in controlled hypertensive individuals. Revista Brasileira de Medicina do Esporte. 2005; 11: 337-340.

30. Simão R, Fleck SJ, Polito M, Monteiro W, Farinatti P. Effects of resistance training intensity, volume, and session format on the postexercise hypotensive response. J Strength Conditioning Res. 2005; 19:853-858.

31. Lizardo JHF, Simões HG. Effects of different resistance exercise sessions on post-exercise hypotension. Braz J Physical Therapy. 2005; 9:249-255.

32. Loenneke JP, Wilson GJ, Wilson JM. A mechanistic approach to blood flow occlusion. Int J Sports Medicine. 2010; 31: 1-4.

33. Manini TM, Clark BC. Blood flow restricted exercise and skeletal muscle health. Exercise Sport Sci Rev. 2009; 37:78-85.

34. Anderson JE, Wozniak AC. Satellite cell activation on fibers: modeling events in vivo-an invited review. Canadian J Physiol Pharmacol. 2004; 82:300-310.
35. Viaro F, Nobre F, Evora PRB. Expression of nitric oxide synthases in the pathophysiology of diseases cardovasculares. Arquivos Brasileiros de Cardiologia. 2000; 74:365-379.

\section{Corresponding author}

Gabriel Rodrigues Neto

Department of Physical Education

Associate Graduate Program in Physical

Education UPE / UFPB

Federal University of Paraíba,

Research Center for Human Movement Sciences

João Pessoa, PB, Brazil.

Email: gabrielrodrigues_1988@hotmail.com

Manuscript accepted on January 23, 2016

Manuscript received on April 14, 2016

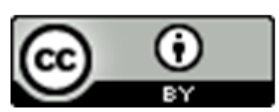

Motriz. The Journal of Physical Education. UNESP. Rio Claro, SP, Brazil - eISSN: 1980-6574 - under a license Creative Commons - Version 3.0 